\title{
LAS NECESIDADES DE I+D+I Y DE FORMACIÓN DE LA INDUSTRIA PRIMARIA DE LA MADERA EN ESPAÑA
}

\author{
Juan I. Fernández-Golfín Seco y Eva Hermoso Prieto \\ INIA-CIFOR. Laboratorios de Maderas. Carretera de la Coruña km 8. 28040-MADRID (España). Correo \\ electrónico: golfin@inia.es, hermoso@inia.es
}

\section{Resumen}

Se presentan algunas recomendaciones de $\mathrm{I}+\mathrm{D}+\mathrm{i}+$ formación para la mejora de la competitividad de los productos de la madera obtenidos a partir de la transformación de maderas nacionales.

Palabras clave: Industria madera, Productos madera, Investigación, Formación, Control de calidad

\section{INTRODUCCIÓN}

Uno de los aspectos que más llama la atención a todos los que trabajan con la madera es que en los últimos años se ha disparado enormemente el número de productos derivados de ella que, tras su caracterización en los laboratorios, acceden al mercado con propiedades y tipologías muy variadas y con adecuación a usos muy diversos.

Junto a la clásica madera aserrada, ahora con formatos, sellos y controles de calidad que hacen de este material un producto mucho más homogéneo que en el pasado, hoy podemos encontrar no sólo productos cuasi-tradicionales como la madera laminada o los tableros de partículas y fibras sino que, además, nos encontramos con un amplísimo elenco de productos mixtos en los que, en algunos casos, es hasta difícil detectar en ellos la presencia de la madera o de sus fibras.

Otro aspecto que se está poniendo en evidencia al observador atento de la realidad de este material, es que la madera cada vez va ganando más adeptos en el mercado de la construcción y el interiorismo y esto es así no sólo porque los nuevos productos se dirigen a aplicaciones específicas en los que su adaptación al uso es casi perfecta sino porque, también, en un entorno de construcción sostenible los valores medioambientales de la madera y sus derivados ganan cada vez más relevancia.

Donde todo esto se pone más en evidencia es en la extraordinaria afluencia de técnicos superiores de la construcción (arquitectos, aparejadores, ingenieros de la construcción, etc.) a las actividades formativas puestas en marcha en el seno del sector (Aulamadera, Maderia, Cátedra de la madera de la Universidad de Navarra y otros impulsos promovidos por entidades industriales o asociativas del sector), lo que nos hace ver con claridad que la madera y sus derivados se están poniendo de moda y que, poco a poco, van ganando adeptos y presencia en el mercado. A este éxito no es ajeno el hecho de que hoy en día el proyectista tiene acceso on-line y gratuito a manuales de productos, de cálculo, de puesta en obra, etc. que le permiten aplicar con sencillez las directrices establecidas en el Código Técnico de la Edificación. Así, por ejemplo, diariamente desde la página web de Maderia (acción formativa e informativa impulsada por empresas del sector de la madera y la construcción con el apoyo de Centros Públicos de investigación como el INIA y el CSIC-Instituto Eduardo Torroja 
de Ciencias de la Construcción) se producen diariamente decenas de descargas de publicaciones y se evacúan no menos consultas sobre el material.

Todo lo anterior nos pone frente a un futuro halagüeño para los productos de la madera, pero... ¿es esto realmente así?.

Respecto de esta cuestión es necesario apuntar que la gran mayoría de los productos de madera más innovadores presentes en el mercado nacional son de importación y fabricados con maderas de fuera de nuestras fronteras, especialmente abeto y picea en el caso de las coníferas y roble, castaño y algunas tropicales en el de las frondosas. Esto nos lleva a extraer una primera conclusión: del próximo incremento en la cuota de mercado de los productos de la madera se beneficiarán fundamentalmente las empresas y los propietarios forestales de fuera de nuestras fronteras junto con los sectores del almacenamiento y la logística nacionales.

Esta, y por todos conocida, primera conclusión nos lleva a una cuestión adicional, como es la de saber las causas de esta preferencia del consumidor y prescriptor nacionales por los productos de importación. Aquí debemos dejar muy clara la que será la segunda conclusión a la que quiero llevar a todos los lectores de estas notas: los niveles de innovación y de implantación de procesos internos de control de la calidad en fábrica y en monte son todavía extraordinariamente bajos a nivel nacional, especialmente si se los compara con los exhibidos por las industrias de la madera y procesos de gestión selvícola del centro y norte de Europa.

Ahondando en el análisis de las preferencias del consumidor llegamos a una penosa tercera conclusión: las maderas nacionales tienen mala imagen en el mercado nacional en gran parte porque se transforman de forma poco eficaz, porque los selvicultores manejan las masas sin integrar en sus procesos de gestión las calidades demandadas por la industria y los usos finales y porque en su conjunto toda la cadena monte-rematantes-industria-intermediarios de mercado final (almacenistas sobre todo) consideran su calidad inadecuada para los usos más innovadores. Esto hace que en España actualmente sean los sectores de los tableros de fragmentación (partículas y fibras), de la pasta y papel y de los embalajes los únicos que tiran de la demanda de madera nacional.
La profunda injusticia que en sí encierra esta tercera conclusión queda puesta claramente de manifiesto analizando la actividad de algunas empresas nacionales que siendo profundamente innovadoras en sus ideas, procesos y productos han sido capaces de poner en el mercado, tanto nacional como internacional, productos de madera altamente innovadores basados en el empleo de maderas nacionales y que compiten en el mercado no sólo por precio sino, también, por calidad. Así es posible ver hoy en día tableros contralaminados, maderas laminadas o dúos y tríos de pino radiata dotados de sellos voluntarios de Marcado CE (por ausencia de norma armonizada) en países tan competitivos para la madera como son Francia y Alemania. Del mismo modo, maderas laminadas de castaño nacional y ciertas elaboraciones nacionales de roble y otras tropicales de importación están ganando crecientes cuotas de mercado fuera de nuestras fronteras. Estos meritorios ejemplos ponen claramente de manifiesto que incorporando innovación, tanto en las ideas como en los procesos, es posible poner en los mercados internacionales producciones nacionales basadas en gran medida en maderas nacionales.

Los asertos del párrafo anterior nos llevan a una, aún más, penosa cuarta conclusión, como es la de que el nivel de innovación y conocimiento de las demandas reales del mercado y las formas para incorporarlas en los procesos de gestión selvícola, de transformación industrial y de logística son alarmantemente bajos en nuestro país.

Por tanto el sector de la madera (el conjunto de la cadena monte-industria) aunque es testigo de cómo, por fin, tiene una normativa que no penaliza el uso de la madera en construcción, cómo de forma creciente los prescriptores del material están formándose a marchas forzadas sobre la mejor forma de usar este material, cómo en nuestra geografía nacional relevantes arquitectos internacionales construyen obras de referencia en madera... sigue manteniendo unos medios de gestión forestal y de producción industrial absolutamente arcaicos que literalmente dejan fuera del mercado a las producciones madereras resultantes de nuestros varios millones de hectáreas de superficie forestal, pensando, quizá y no se en base a qué, que nuestras maderas no valen para "eso". 
Lo curioso de esta situación es que no es en absoluto novedosa y para ello traigo aquí textualmente las palabras del ingeniero de montes $\mathrm{L}$. Lillo Sanz que en 1931 impartió con ocasión del II Congreso Nacional de la Madera y de las industrias derivadas (8 al 18 de octubre de 1931):

La influencia del monte en la Economía de un país es decisiva, sobre todo cuando su territorio es tan quebrado como el nuestro, y de aquí la necesidad de someterlo a una producción intensiva y de que sus productos se transformen según métodos modernos.

Nuestras fábricas forestales, en general sin conexión con el monte de donde han de recibir las primeras materias, lucha con desventaja en el mercado nacional con la producción extranjera, y no llenan el papel que tales industrias deben cumplir en el desenvolvimiento de nuestra Economía.

Se impone, pues, la revisión de estas industrias, ya que su prosperidad es punto capital para que la extensa superficie forestal de nuestro país, hoy desarbolada, sea restaurada; ningún estímulo mejor para repoblar terrenos rasos que una renta elevada en los suelos arbolados, y para que éstos la perciban es necesario que sus productos sean aprovechados íntegramente y transformados industrialmente en los que el mercado reclama.

Un estudio de las industrias forestales españolas nos muestra que los grandes aserraderos no están precisamente cerca de las masas de monte, sino que se sitúan en los puertos con vistas a la madera extranjera; las fábricas resineras y las que transforman el corcho, más que unirse a la propiedad forestal, tienden a separarse de ella, y hasta a hacer antagónicos sus intereses; tampoco se unen entre si estas fábricas, a pesar de tratarse de industrias de exportación, tan necesitadas de sostener en el Extranjero el precio de sus productos frente a naciones mejor organizadas comercialmente que la nuestra.

En cuanto a las papeleras, éstas reciben del Extranjero toda la madera y todas las pastas que emplean en la fabricación del papel, y es industria, por tanto, que depende del exterior, a pesar de que en España podría encontrar medios de abastecerse.
Se impone, pues, un cambio en la orientación hasta ahora seguida, y que entre el monte y las industrias que utilizan sus productos se establezca la relación debida, que las fábricas forestales españolas utilicen los productos de nuestros montes.

Ante la lectura de estas palabras nos preguntamos sobre si tras casi 100 años hemos sido capaces de subvertir de forma significativa la negativa situación que en nuestros montes y sus gentes ya se apreciaba a comienzos del siglo pasado.

Para ser totalmente justos en el análisis es necesario apuntar que algunas de las industrias nacionales de la madera, como son todas las de los tableros de desintegración (partículas y fibras) así como las de la celulosa, desde hace tiempo que son totalmente competitivas a nivel internacional, motivo por lo cual el problema de falta de competitividad industrial se centra casi exclusivamente en la industria de la primera transformación. Es cierto, sin embargo, que aunque las industrias de la desintegración y de la celulosa así como las de primera transformación focalizadas en el mercado del embalaje hacen uso habitual de las maderas nacionales, no ocurre lo mismo con el resto de industrias de la transformación, de manera que los productos más innovadores y rentables destinados a los mercados de la decoración y la construcción siguen siendo fabricados con maderas foráneas, en su mayoría producidos por empresas también foráneas.

En estas tierras vascas, tan cercanas para mi tanto desde el ámbito familiar como profesional, los avatares del pino radiata ponen claramente de manifiesto la realidad de lo que más arriba he apuntado. Vemos como esta especie abastece regularmente a las industrias del tablero, la celulosa o el embalaje pero es pobremente utilizada por las industrias del aserrado y de la segunda transformación orientadas hacia la elaboración de elementos estructurales. Apreciamos como a todo lo largo y ancho del territorio del País Vasco se ven obras de edificación y construcción civil relevantes, hechas en su gran mayoría en abeto, obras que por su pobre nivel de durabilidad conferida (la madera de abeto y picea no son capaces de recibir eficazmente los tratamientos que las protejan de la intemperie, cosa que no pasa con el pino radiata) aportan mala imagen a la madera y que si se hubieran construido con madera de pino radiata bien elaborada y correctamente tratada en función de las clases de 
uso estarían hoy en día con perfecto estado de conservación, transmitiendo a esta madera en particular y a todo el sector una imagen mucho más adaptada a la realidad que vemos en otros territorios y países. En este sentido llama poderosísimamente la atención como el primer puente de madera de pino radiata se construyó en la autovía A-7, en el Ayuntamiento de Sneek (Holanda) y no en una zona tan productora de esta madera, como es el País Vasco. Para los conocedores y amantes de esta madera es doloroso comprobar cómo incluso desde la propia Administración autonómica se ha fomentado en sus proyectos el uso de maderas foráneas quizá pensando que de ese modo fomentaban la calidad de sus obras, sin reparar que con ello no sólo infringían un daño notable a sus propietarios forestales sino que, también, lo hacían con respecto a la calidad de sus realizaciones. El miedo a la madera está, desgraciadamente, muy extendido y se palía de forma equivocada empleando productos de importación, pensando de ese modo que se adquiere una cierta seguridad en el resultado. Lamentable error de juicio, porque cuando las cosas vienen mal dadas para la madera son, precisamente, las maderas tan permeables como el pino radiata las que convenientemente tratadas pueden aportar la durabilidad adecuada.

Por todo ello y en aras a ayudar a mejorar la situación del sector industrial y por extensión del forestal, centraré el análisis de las necesidades de $\mathrm{I}+\mathrm{D}+\mathrm{i}$ y de formación en el ámbito de la producción primaria y de sus materias primas, de acuerdo con el siguiente esquema:

1. Análisis de las necesidades que gravitan sobre la materia prima

2. Análisis de las necesidades que gravitan sobre los procesos de transformación

3. Análisis de las necesidades que gravitan sobre los productos finales y su adaptación al uso

4. Análisis de las necesidades que gravitan sobre la caracterización ambiental de materias primas, productos y sistemas en base madera

\section{ANÁLISIS DE LAS NECESIDADES QUE GRAVITAN SOBRE LA MATERIA PRIMA FORESTAL}

Un aspecto sobre el que todos estaremos de acuerdo es que un industrial puede arruinar una buena calidad de materia prima con un procesamiento erróneo pero difícilmente puede mejorar la calidad de la materia prima que le dan. Por dicho motivo el control sobre la calidad de los suministros es una de las primeras medidas que todo industrial debe llevar a cabo para introducir una mejora significativa en sus productos y procesos.

\section{Diseño de sistemas eficaces para la evaluación de la calidad (en pie y apeada) de la producción forestal \\ Métodos visuales}

Conscientes de este problema, durante muchos años el Comité Europeo de Normalización CEN TC 175 ha venido trabajando sobre metodologías de tipo visual aplicables en monte para la clasificación de la madera en rollo apeada. En este campo podemos citar las siguientes normas:

- UNE-EN 844-1:1996. Madera aserrada y madera en rollo. Terminología. Parte 1: Términos generales comunes a la madera aserrada y a la madera en rollo.

- UNE-EN 844-2:1997. Madera aserrada y madera en rollo. Terminología. Parte 2: Términos generales relativos a la madera en rollo.

- UNE-EN 844-5:1997. Madera aserrada y madera en rollo. Terminología. Parte 5: Términos relativos a las dimensiones de la madera en rollo.

- UNE-EN 1309-2:2007. Madera aserrada y madera en rollo. Método de medida de las dimensiones. Parte 2: Madera en rollo. Requisitos para la medida y reglas de cálculo del volumen.

- UNE-EN 1310:1997. Madera aserrada y madera en rollo. Método de medida de las singularidades.

- UNE-EN 1316-1:2012. Madera en rollo de frondosas: Robles y haya (la anterior era de 1997)

- UNE-EN 1316-1:2012. Madera en rollo de frondosas: Chopo (la anterior era de 1997)

- UNE-EN 1927-1:2008. Clasificación de calidad de la madera en rollo de coníferas. Parte 1: Piceas y abetos.

- UNE-EN 1927-2:2008. Clasificación de calidad de la madera en rollo de coníferas. Parte 2: Pinos. 
- UNE-EN 1927-3:2008. Clasificación de calidad de la madera en rollo de coníferas. Parte 3: Alerces y abetos de Douglas.

Este amplio elenco de normas, ya en aplicación desde hace años en el sector forestal europeo, resulta de urgente empleo y adaptación por parte del sector forestal nacional para la correcta definición de la oferta de madera en rollo derivada de la producción forestal.

No existen, y es de urgente necesidad, sistemas visuales para la clasificación de la madera en pie, sistemas que serían de utilidad no sólo para los industriales y rematantes sino, también, para los propios gestores forestales ya que les permitiría evaluar la incidencia de sus labores selvícolas o mejoramiento genético.

\section{Métodos no destructivos de tipo automatizado}

En la actualidad existen diversos grupos de investigación que trabajan en la evaluación no destructiva de la calidad, incluso en pie, mediante métodos sónicos de diversa naturaleza o, incluso, mediante sistemas basados en radiaciones ionizantes instalados en los parques de madera en rollo de las plantas de procesamiento. Este camino es interesante y debe ser explorado pero es criterio de este autor que es más urgente la puesta en marcha de los sistemas de evaluación visual contenidos en las normas anteriormente mencionadas y que ya están en funcionamiento en el sector forestal europeo, lo que permitiría abrir la oferta de producción forestal al resto del entorno europeo e incorporar la terminología internacional.

Para especies como el pino radiata o el castaño resulta urgente la puesta a punto de sistemas y protocolos para la detección (o la obtención de indicadores de todo tipo que permitan prever su presencia) de acebolladuras en pie o en las rollas recién apeadas.

\section{Análisis del efecto de determinadas prácticas selvícolas en la calidad de la madera}

En concreto el sector industrial está preocupado especialmente por la presencia de los siguientes defectos:

\section{Coníferas}

- Madera juvenil (especialmente $P$. radiata y P. laricio)

- Acebolladuras (especialmente $P$. radiata)

- Textura irregular (afecta sobre todo a la chapa)
- Enteamiento (P. laricio)

- Excentricidad

- Nudosidad excesiva

Frondosas

- Desviación de fibras (especialmente castaño)

- Tensiones de crecimiento (Eucalipto)

- Acebolladuras (especialmente castaño)

- Textura irregular

\section{Análisis de la influencia de la genética en la calidad de la madera}

Cuando estás acostumbrado a clasificar maderas no es infrecuente comprobar cómo determinados árboles presentan tasas de rechazo cercanas al $100 \%$ mientras que otros procedentes de la misma zona presentan rendimientos máximos en madera clasificada, señalando que puede existir alguna influencia de tipo genético que no se ha evaluado correctamente en las labores de policía.

Esto es especialmente patente, por ejemplo, con la desviación de fibras en el pino laricio o con la textura (especialmente con la anchura de la madera de verano dentro del anillo) dentro del pino radiata o el pino pinaster. La presencia, y su cuantía, de madera juvenil es algo que también parece tener influencia genética junto con la mayor o menor longitud de fibra y la homogeneidad de su depósito dentro del anillo de crecimiento.

Es por dicho motivo por el que en muchas ocasiones a igualdad de aspectos morfológicos externos determinados individuos o determinadas procedencias manifiestan rendimientos cualitativos muy diferentes, haciendo muy difícil al industrial prever el rendimiento económico derivado de su aprovechamiento industrial.

Los industriales nos quejamos de que en muchos casos los indicadores de calidad que se emplean en la evaluación de la mejora genética no reflejan suficientemente bien los aspectos que realmente inciden en la calidad de la madera desde un punto de vista industrial.

\section{ANÁLISIS DE LAS NECESIDADES QUE GRAVITAN SOBRE LOS PROCESOS DE TRANSFORMACIÓN}

Los procesos de fabricación que están más necesitados de innovación y formación (transferencia tecnológica) son los siguientes: 
- En el aserrado sistemas de optimización del corte de la madera según demanda

- En el aserrado sistemas de clasificación en línea de la madera aserrada por criterios de resistencia y aspecto.

- En el secado sistemas de optimización energética de los procesos, en especial los referidos al secado de maderas de gruesas escuadrías.

- En el tratamiento de la madera para la mejora de su durabilidad frente a los agentes de la degradación

- Puesta a punto para las maderas españolas de sistemas de tratamiento de bajo impacto ambiental

- Acetilación y furfurilación

- Térmicos (especialmente los basados en el uso del vapor)

- Identificación de los factores que influyen en la durabilidad a largo plazo de las distintas maderas

- Incorporación de la nanotecnología para la reducción de la lixiviación de los tratamientos químicos y la mejora de su comportamiento ante el fuego.

- En el tratamiento fitosanitario de la madera para su uso en la elaboración de embalajes de exportación es necesario no sólo optimizar el empleo de la norma internacional NIMF-15 sino, además, evaluar si los actuales tratamientos antiazulado son eficaces frente al nematodo y Fusarium.

- En el encolado de la madera, mejora de los procesos de encolado para productos laminados destinados a usos de exterior.

- Caracterización energética de los procesos de fabricación con vistas a su optimización y su empleo en las declaraciones ambientales de productos.

\section{ANÁLISIS DE LAS NECESIDADES QUE GRAVITAN SOBRE LOS PRODUCTOS FINALES Y SU ADAPTACIÓN AL USO}

El prestigio de los productos nacionales sólo puede ser adquirido a través de su perfecta adecuación al uso, la completa documentación asociada al producto y la existencia de recomendaciones claras y eficaces respecto de su almacenamiento, puesta en obra y mantenimiento.
A este respecto es necesario tomar en cuenta el hecho de que para los usos de interior (Clase de uso 1) y semiexterior (Clase de uso 2) los productos de la madera sólo presentan comportamientos no adecuados cuando se dan circunstancias extraordinarias no previstas inicialmente (errores de almacenamiento, de puesta en obra, escapes de agua, falta de mantenimiento de elementos de cubrición e impermeabilización, etc.) y que es en los usos de exterior (especialmente en los incluidos dentro de la Clase de uso 3 en edificación y los asignables a Clase de uso 4 en construcción civil) donde se dan las mayores irregularidades, todas ellas derivadas de una falta de adecuación de la durabilidad del producto (natural o conferida mediante tratamiento) a las amenazas derivadas de las condiciones de empleo del mismo.

Por dicho motivo y considerando que la norma europea EN 335 de Clases de Uso no es sino una norma marco que no deja, tanto para el prescriptor como para el industrial, perfectamente claros los criterios de asignación de las clases, es necesario establecer pautas para llevar a cabo esta tarea, pautas que deben derivarse de un profundo análisis de todos los factores (climáticos, geográficos, de diseño, etc.) que afectan al comportamiento y la forma más eficaz de inhibir o posponer su actuación. Sólo prescribiendo adecuadamente las maderas y los tratamientos más adecuados se puede asegurar una perfecta durabilidad de los productos y obras, lo que tendrá no sólo incidencia en la imagen que los usuarios y prescriptores tengan respecto de los productos de la madera sino, también en la evaluación ambiental de los edificios y obras civiles con ellos construidos.

En este ámbito volvemos a destacar la importancia de adecuar la calidad del encolado a las exigencias derivadas físico-mecánicas del uso, lo que plantea la necesidad de establecer pautas también para la adecuada asignación de las distintas Clases de Servicio (Eurocódigo 5 y Código Técnico de la Edificación) por parte de los proyectistas y el diseño e implantación de sistemas de evaluación de la calidad del encolado por clase de servicio por parte de los industriales.

Además de lo anterior, el sector de la industria de la madera debe ser perfecto conocedor de todas las demandas de calidad que gravitan sobre sus productos, tanto normativas (Marcado CE, Directivas diversas, Código Técnico de la 
Edificación, REACH, etc.) como del mercado. Para ello el que esto escribe considera fundamentales las siguientes acciones:

- Implantación de sistemas eficaces de Control de la Producción en Fábrica que permitan una rápida detección de las no conformidades en el seno de las propias empresas, evitando con ello la salida al mercado de productos de calidad insuficiente.

- Implantación de círculos de calidad a nivel de producto, que permitan identificar los problemas y detectar con rapidez las necesidades de los consumidores y usuarios.

- Implantación de sistemas de formación y capacitación permanente de clasificadores de productos de la construcción (fundamentalmente madera aserrada).

- Implantación del Marcado CE tanto en los productos de obligatoria presencia (por presencia de norma armonizada) como en los de carácter voluntario (a través de DITEs y DEEs).

- Implantación de sistemas de certificación de cadena de custodia, como paso obligado para la posterior emisión de Declaraciones ambientales de sus productos.

\section{ANÁLISIS DE LAS NECESIDADES QUE GRAVITAN SOBRE LA CARACTERIZACIÓN AMBIENTAL DE MATERIAS PRIMAS, PRODUCTOS Y SISTEMAS EN BASE MADERA}

Como ya se puso de manifiesto en la introducción, los valores ambientales de los productos de la madera tiran fuertemente de ellos en entornos de mercado en los que, como en la construcción, la sostenibilidad ambiental es un criterio en alza.

Los productores nacionales, si quieren competir en este mercado ambiental en alza, deben ser capaces de incorporar en sus productos declaraciones ambientales que permitan a los consumidores tener la información necesaria para elegirlos a ellos con preferencia a otros con índices ambientales más modestos. La implantación de los criterios ambientales a nivel de producto obliga, pero también permite, a los industriales avanzar en la senda de la optimización de los diseños, de los procesos de fabricación y de la adaptación al uso, toda vez que no hay elemento que distorsione más las evaluaciones de "cuna a tumba" de edificios y obras civiles que la necesidad de reiteradas operaciones de reparación y sustitución por deficiente durabilidad (tomado este concepto en su sentido más amplio).

Para urgir al industrial a incorporar sus deberes ambientales en su gestión empresarial basta decir que el Reglamento de Productos de la Construcción (que entra definitivamente en vigor el 13/7/2013), en su considerando 56 establece textualmente lo siguiente "Para la evaluación del uso sostenible de los recursos y el impacto medioambiental de las obras de construcción deben utilizarse, cuando estén disponibles, las declaraciones medioambientales de productos". Ya se trabaja en el seno del CEN para introducir en las revisiones de las normas armonizadas estos aspectos con vista a su incorporación al Marcado CE obligatorio para los productos de la construcción con norma armonizada.

Ahora bien, los deberes ambientales no sólo afectan a los industriales sino al sector forestal en su conjunto ya que al hacer valer los productos de la madera una huella de carbono de tipo negativo (sumidero) es necesario asegurar que estos productos proceden de aprovechamientos sostenibles. De hecho, los proyectos de normas europeas prEN 16449 (de cálculo del $\mathrm{CO}_{2}$ secuestrado en los productos de la madera) y prEN 16485 (de Reglas de Categoría para los productos de la madera), están siendo fuertemente contestadas por los sectores del acero y el hormigón en base a la existencia de aprovechamientos forestales de tipo no sostenible.

De acuerdo con lo anterior parece claro que la valoración y posterior declaración ambiental de los productos de la madera debe efectuarse únicamente en un entorno de acreditación de la sostenibilidad del conjunto de la cadena monteindustria (lo que implica la acreditación no sólo de la GFS sino de la cadena de custodia también). Dado que la declaración ambiental de un producto final ha de basarse no sólo en sus propios procesos de transformación sino en la valoración de las materias primas, es necesario que el conjunto del sector forestal efectúe un análisis (y mejora) de sus procesos bajo la óptica de los impactos ambientales producidos.

Debe tomar muy en cuenta el gestor forestal que estas demandas ambientales son una extraor- 
dinaria oportunidad para el mercado de las maderas nacionales ya que al ser los productos de la madera de baja densidad, los impactos ambientales derivados del transporte pesan mucho en la valoración ambiental final de los productos, lo que hace más competitivos ambientalmente a los productos producidos en las proximidades de su empleo.

Pero existe un aspecto final que no quiero dejar pasar pues es uno de los grandes valores de la madera: la posibilidad de su aprovechamiento energético y reutilización.

Uno de los aspectos que diferencian, por ejemplo, a la madera de sus competidores en el ámbito de los productos de la construcción es la posibilidad de valorizar energéticamente o de reutilizar los subproductos que se producen en cada etapa de sus procesos de fabricación y puesta en obra. De ahí que ahora se entienda mejor la absoluta necesidad que tenemos de optimizar los diseños y usos como medio de minimizar los tratamientos químicos que no hacen sino imposibilitar el uso libre de los subproductos, lo que pesa de forma negativa en las evaluaciones ambientales de "cuna a tumba".
Respecto de la valorización energética de los residuos en cualquier fase del proceso productivo y montaje, es necesario optimizar los métodos y productos (granel, briquetas, pellets, etc.), toda vez que, por ejemplo, la incorporación de sistemas de calefacción basados en la biomasa afecta de forma muy positiva a la valoración ambiental global de los edificios, efectuada en el conjunto de toda su vida en servicio.

La acreditación y optimización del mix energético en el seno de nuestras industrias es algo que, también, pesa de forma enormemente positiva en la Declaración ambiental de nuestros productos, motivo por lo cual es necesario trabajar con intensidad en este campo.

De todo lo dicho hasta ahora se desprende que en el futuro más inmediato se abre un conjunto de posibilidades para el despegue sostenible de nuestro sector y que es obligación de todos aplicar la mayor inteligencia y tesón posibles para conseguir subvertir la situación y permitir que, esta vez sí, las palabras de L. Lillo en el II Congreso Nacional de la Madera de 1931 no sigan siendo vigentes por mucho más tiempo. 